\title{
SOME INEQUALITIES OF ALGEBRAIC POLYNOMIALS
}

\author{
A. K. VARMA \\ (Communicated by Andrew Bruckner) \\ Dedicated to Professor A. Sharma and Mrs. Durga Sharma
}

\begin{abstract}
Erdös and Lorentz showed that by considering the special kind of the polynomials better bounds for the derivative are possible. Let us denote by $H_{n}$ the set of all polynomials whose degree is $n$ and whose zeros are real and lie inside $[-1,1)$. Let $P_{n} \in H_{n}$ and $P_{n}(1)=1$; then the object of Theorem 1 is to obtain the best lower bound of the expression $\int_{-1}^{1}\left|P_{n}^{\prime}(x)\right|^{p} d x$ for $p \geq 1$ and characterize the polynomial which achieves this lower bound. Next, we say that $P_{n} \in S_{n}[0, \infty)$ if $P_{n}$ is a polynomial whose degree is $n$ and whose roots are all real and do not lie inside $[0, \infty)$. In Theorem 2, we shall prove Markovtype inequality for such a class of polynomials belonging to $S_{n}[0, \infty)$ in the weighted $L_{p}$ norm $\left(p\right.$ integer). Here $\left\|P_{n}\right\|_{L_{p}}=\left(\int_{0}^{\infty}\left|P_{n}(x)\right|^{p} e^{-x} d x\right)^{1 / p}$. In Theorem 3 we shall consider another analogous problem as in Theorem 2.
\end{abstract}

\section{INTRODUCTION}

Let $H_{n}$ be the set of all polynomials whose degree is $n$ and whose zeros are real and lie inside $[-1,1)$. Concerning this class of polynomials belonging to $H_{n}$ we shall prove the following theorem.

Theorem 1. Let $P_{n} \in H_{n}$, subject to the condition $P_{n}(1)=1$. Then we have (for $p \geq 1)$

$$
\int_{-1}^{1}\left|P_{n}^{\prime}(x)\right|^{p} d x \geq \frac{n^{p}}{2^{p-1}((n-1) p+1)},
$$

with equality iff $P_{n}(x)=\left(\frac{1+x}{2}\right)^{n}$.

The case $p=2$ was considered in [5] and [8].

In 1964 G. Szegö [6] studied the order of magnitude of $\left\|P_{n}^{\prime}\right\|_{L_{\infty}} /\left\|P_{n}\right\|_{L_{\infty}}$ for unrestricted polynomials $P_{n}$ of degree $\leq n$ for the norm

$$
\left\|P_{n}\right\|=\sup _{x \geq 0}\left|P_{n}(x) e^{-x}\right|
$$

on $(0, \infty)$. More precisely, he proved the following

Received by the editors April 30,1993 and, in revised form, July 6, 1993 and August 10, 1993. 1991 Mathematics Subject Classification. Primary 26D15.

The author is deceased (December 8, 1994). 
Theorem A. Let $P_{n}(x)$ be a polynomial of fixed degree $n$ and not vanishing identically. Then we have

$$
\left\|P_{n}^{\prime}\right\|<c n\left\|P_{n}\right\|, \quad n=2,3, \ldots .
$$

In 1968, G. G. Lorentz [4] considered the problem of G. Szegö for the special polynomials with positive coefficients in $x$

$$
P_{n}(x)=\sum_{k=0}^{n} a_{k} x^{k}, \quad a_{k} \geq 0, k=0,1, \ldots, n
$$

and where the norm of a function $P_{n}$ on $(0, \infty)$ is given by

$$
\left\|P_{n}\right\|=\sup _{x \geq 0}\left|P_{n}(x) e^{-w(x)}\right|
$$

where $w(x)$ increases on $(0, \infty)$.

Motivated by the theorems of G. Szegö [6] and Lorentz [4] and the earlier result of the author [9] we shall consider the following problem concerning the class of polynomials $P \in S_{n}[0, \infty)$. Here let $S_{n}[0, \infty)$ be the set of all polynomials whose degree is $n$ and whose roots are real and do not lie inside $[0, \infty)$. It is easy to see that if $P_{n} \in S_{n}[0, \infty)$, then it can be expressed in the form

$$
P_{n}(x)=\sum_{k=0}^{n} a_{k} x^{k}, \quad a_{k} \geq 0 \text { for } k=0,1, \ldots, n .
$$

Now, we state the following.

Theorem 2. Let

$$
P_{n}(x)=\sum_{k=0}^{n} a_{k} x^{k}, \quad a_{k} \geq 0 \text { for } k=0,1, \ldots, n .
$$

Then, we have for any positive integer $p$

$$
\frac{\int_{0}^{\infty}\left|P_{n}^{\prime}(x)\right|^{p} e^{-x} d x}{\int_{0}^{\infty}\left|P_{n}(x)\right|^{p} e^{-x} d x} \leq \frac{1}{p !}
$$

with equality iff $P_{n}(x)=\alpha x$.

It is of some interest to remark that the extreme value in the above inequality is independent of the degree of the polynomials. In view of the above theorem, we shall now prove

Theorem 3. Let $P_{n} \in S_{n}[0, \infty)$ and $r, p$ be positive integers. Then we have (for $r \leq p)$

$$
\int_{0}^{\infty}\left|P_{n}^{\prime}(x)\right|^{r} x^{p-1} e^{-x} d x \leq \frac{n^{r}(n r+p-r-1) !}{(n r+p-1) !} \int_{0}^{\infty}\left|P_{n}(x)\right|^{r} x^{p-1} e^{-x} d x
$$

with equality iff $P_{n}(x)=\alpha x^{n}$.

\section{Proof of TheOREM 1}

Let $P_{n} \in H_{n}, P_{n}(1)=1$. We shall denote the zeros of $P_{n}(x)$ by $x_{n}, x_{n-1}$, $\ldots, x_{2}, x_{1}$ satisfying the inequality

$$
-1 \leq x_{n} \leq x_{n-1} \leq \cdots \leq x_{2} \leq x_{1}<1 .
$$


We may express $P_{n}(x)$ by

$$
P_{n}(x)=c \prod_{i=1}^{n}\left(x-x_{i}\right), \quad P_{n}(1)=1 .
$$

Next, we note that

$$
P_{n}(x) \geq 0, \quad x_{1} \leq x \leq 1 .
$$

From (2.1)-(2.3) and

$$
P_{n}^{\prime}(x)=P_{n}(x) \sum_{i=1}^{n} \frac{1}{x-x_{i}}
$$

we obtain

$$
P_{n}^{\prime}(x) \geq 0, \quad x_{1} \leq x \leq 1 .
$$

Next, we note that for $y \geq 0$ and $p \geq 1$ we have

$$
y^{p}-1 \geq p(y-1)
$$

with equality only for $y=1$ or for $p=1$. Proof of (2.6) can be given as follows. Consider $(y \geq 0, p \geq 1) \varphi(y)=y^{p}-1-p(y-1)$. Then $\varphi(1)=$ $0, \varphi^{\prime}(1)=0, \varphi^{\prime \prime}(y)=p(p-1) y^{p-2} \geq 0$. Therefore, by using Taylor's Theorem, we have

$$
\begin{aligned}
\varphi(y) & =\varphi(1)+(y-1) \varphi^{\prime}(1)+\varphi^{\prime \prime}(\xi) \frac{(y-1)^{2}}{2 !} \\
& =\varphi^{\prime \prime}(\xi) \frac{(y-1)^{2}}{2 !}=\frac{p(p-1) \xi^{p-2}(y-1)^{2}}{2} \geq 0
\end{aligned}
$$

( $\xi$ being between $y$ and 1 ).

From this (2.6) follows. Next, we put

$$
y=\frac{P_{n}^{\prime}(x)}{\frac{n P_{n}(x)}{x-x_{n}}}
$$

in (2.6). Then we have $\left(x_{1} \leq x \leq 1\right)$ after some simplification

$$
\begin{aligned}
\left(P_{n}^{\prime}(x)\right)^{p} \geq & \frac{p n^{p-1} P_{n}^{\prime}(x)\left(P_{n}(x)\right)^{p-1}}{\left(x-x_{n}\right)^{p-1}} \\
& -(p-1) n^{p} \frac{\left(P_{n}(x)\right)^{p}}{\left(x-x_{n}\right)^{p}}
\end{aligned}
$$

Clearly, then

$$
\begin{array}{r}
\int_{x_{1}}^{1}\left|P_{n}^{\prime}(x)\right|^{p} d x \geq p n^{p-1} \int_{x_{1}}^{1} \frac{P_{n}^{\prime}(x)\left(P_{n}(x)\right)^{p-1}}{\left(x-x_{n}\right)^{p-1}} d x \\
-(p-1) n^{p} \int_{x_{1}}^{1} \frac{\left(P_{n}(x)\right)^{p}}{\left(x-x_{n}\right)^{p}} d x .
\end{array}
$$

Next, we note that

$$
\begin{gathered}
p \int_{x_{1}}^{1} \frac{P_{n}^{\prime}(x)\left(P_{n}(x)\right)^{p-1}}{\left(x-x_{n}\right)^{p-1}} d x=\int_{x_{1}}^{1}\left(\frac{d}{d x}\left(P_{n}(x)\right)^{p}\right) \frac{1}{\left(x-x_{n}\right)^{p-1}} d x \\
=\frac{\left(P_{n}(1)\right)^{p}}{\left(1-x_{n}\right)^{p-1}}+(p-1) \int_{x_{1}}^{1} \frac{\left(P_{n}(x)\right)^{p}}{\left(x-x_{n}\right)^{p}} d x .
\end{gathered}
$$


Therefore, from (2.7) and above we obtain

$$
\begin{aligned}
\int_{x_{1}}^{1}\left|P_{n}^{\prime}(x)\right|^{p} d x \geq & n^{p-1}\left\{\frac{1}{\left(1-x_{n}\right)^{p-1}}+(p-1) \int_{x_{1}}^{1} \frac{\left(P_{n}(x)\right)^{p}}{\left(x-x_{n}\right)^{p}} d x\right\} \\
& -(p-1) n^{p} \int_{x_{1}}^{1} \frac{\left(P_{n}(x)\right)^{p}}{\left(x-x_{n}\right)^{p}} d x \\
\geq & \frac{n^{p-1}}{\left(1-x_{n}\right)^{p-1}}-n^{p-1}(n-1)(p-1) \int_{x_{1}}^{1} \frac{\left(P_{n}(x)\right)^{p}}{\left(x-x_{n}\right)^{p}} d x .
\end{aligned}
$$

Since

$$
0 \leq x-x_{k} \leq x-x_{n}, \quad k=1,2, \ldots, n, \quad x_{1} \leq x \leq 1,
$$

we have

$$
\begin{aligned}
\left(P_{n}^{\prime}(x)\right)^{p} & =\left(P_{n}(x)\right)^{p}\left(\sum_{k=1}^{n} \frac{1}{x-x_{k}}\right)^{p} \\
& \geq \frac{n^{p}\left(P_{n}(x)\right)^{p}}{\left(x-x_{n}\right)^{p}}, \quad x_{1} \leq x \leq 1 .
\end{aligned}
$$

From above and (2.8) we obtain

$$
n^{p} \int_{x_{1}}^{1} \frac{\left(P_{n}(x)\right)^{p}}{\left(x-x_{n}\right)^{p}} d x \leq \int_{x_{1}}^{1}\left(P_{n}^{\prime}(x)\right)^{p} d x
$$

and

$$
\int_{x_{1}}^{1}\left(P_{n}^{\prime}(x)\right)^{p} d x \geq \frac{n^{p-1}}{\left(1-x_{n}\right)^{p-1}}-\frac{(n-1)(p-1)}{n} \int_{x_{1}}^{1}\left(P_{n}^{\prime}(x)\right)^{p} d x
$$

with equality iff $P_{n}^{\prime}(x)=\frac{n P_{n}(x)}{x-x_{n}}$ and $x_{n}=-1, p \geq 1$.

From the above (1.1) follows. Thus, we have proved Theorem 1.

\section{Proof of Theorem 2}

We set

$$
P_{n}(x)=\sum_{k=0}^{n} a_{k} x^{k}, \quad a_{k} \geq 0, k=0,1, \ldots, n,
$$

and note that $P_{n}^{(r)}(x)$ is a polynomial of degree $\leq n-r$ in $x$ with nonnegative coefficients. If we denote

$$
P_{n}(x)=a_{0}+r_{n}(x), \quad a_{0} \geq 0, r_{n}(x)=\sum_{k=1}^{n} a_{k} x^{k}, \quad a_{k} \geq 0,
$$

then we notice that

$$
\frac{\int_{0}^{\infty}\left(P_{n}^{\prime}(x)\right)^{p} e^{-x} d x}{\int_{0}^{\infty}\left(P_{n}(x)\right)^{p} e^{-x} d x} \leq \frac{\int_{0}^{\infty}\left(r_{n}^{\prime}(x)\right)^{p} e^{-x} d x}{\int_{0}^{\infty}\left(r_{n}(x)\right)^{p} e^{-x} d x} .
$$

Therefore, in order to prove Theorem 2 it is enough to consider the class of all polynomials $P_{n}(x)$ of degree $\leq n$ in $x$ with nonnegative coefficients 
subject to the condition that $P_{n}(0)=0$. Next, we note that $P_{n}^{(r)}(x) \geq 0$, for $0 \leq x<\infty$, and

$$
\begin{aligned}
\int_{0}^{\infty} & \left(P_{n}^{\prime}(x)\right)^{p-r}\left(P_{n}(x)\right)^{r} e^{-x} d x \\
& =\int_{0}^{\infty} P_{n}^{\prime}(x)\left(P_{n}^{\prime}(x)\right)^{p-r-1}\left(P_{n}(x)\right)^{r} e^{-x} d x \\
& =-\int_{0}^{\infty} P_{n}(x)\left[-\left(P_{n}^{\prime}(x)\right)^{p-r-1}\left(P_{n}(x)\right)^{r} e^{-x}+r\left(P_{n}(x)\right)^{r-1}\left(P_{n}^{\prime}(x)\right)^{p-r} e^{-x}\right. \\
& \left.+(p-r-1)\left(P_{n}^{\prime}(x)\right)^{p-r-2} P_{n}^{\prime \prime}(x)\left(P_{n}(x)\right)^{r} e^{-x}\right] d x .
\end{aligned}
$$

From above, we may conclude that

$$
\begin{aligned}
(r+1) & \int_{0}^{\infty}\left(P_{n}^{\prime}(x)\right)^{p-r}\left(P_{n}(x)\right)^{r} e^{-x} d x \\
= & \int_{0}^{\infty}\left(P_{n}^{\prime}(x)\right)^{p-r-1}\left(P_{n}(x)\right)^{r+1} e^{-x} d x \\
& +(r+1-p) \int_{0}^{\infty}\left(P_{n}^{\prime}(x)\right)^{p-r-2} P_{n}^{\prime \prime}(x)\left(P_{n}(x)\right)^{r} e^{-x} d x \\
& \leq \int_{0}^{\infty}\left(P_{n}^{\prime}(x)\right)^{p-r-1}\left(P_{n}(x)\right)^{r+1} e^{-x} d x \quad(p \geq r+1)
\end{aligned}
$$

with equality iff $P_{n}(0)=0$ and $P_{n}^{\prime \prime}(x)=0$.

Putting $r=0,1, \ldots, p-1$ we obtain

$$
\int_{0}^{\infty}\left(P_{n}^{\prime}(x)\right)^{p} e^{-x} d x \leq \frac{1}{p !} \int_{0}^{\infty}\left(P_{n}(x)\right)^{p} e^{-x} d x
$$

with equality iff $P_{n}^{\prime \prime}(x)=0$ and $P_{n}(0)=0$.

From this the proof of Theorem 2 is complete.

\section{Proof of Theorem 3}

Let $x_{1}, x_{2}, \ldots, x_{n}$ be any real zero of $P_{n} \in S_{n}[0, \infty)$. Then $x_{k} \leq 0, k=$ $1,2, \ldots, n$. Also, using Turán's identity [7] we have

$$
\left(P_{n}^{\prime}(x)\right)^{2}-P_{n}(x) P_{n}^{\prime \prime}(x)=\left(P_{n}(x)\right)^{2} \sum_{k=1}^{n} \frac{1}{\left(x-x_{k}\right)^{2}} \text {. }
$$

Therefore we obtain

$$
\begin{aligned}
x\left[\left(P_{n}^{\prime}(x)\right)^{2}-P_{n}(x) P_{n}^{\prime \prime}(x)\right] & =P_{n}^{2}(x) \sum_{k=1}^{n} \frac{x-x_{k}+x_{k}}{\left(x-x_{k}\right)^{2}} \\
& \leq P_{n}^{2}(x) \sum_{k=1}^{n} \frac{1}{x-x_{k}}=P_{n}(x) P_{n}^{\prime}(x) .
\end{aligned}
$$

Since $P_{n} \in S_{n}[0, \infty)$, it follows that $P_{n}^{(r)}(x) \geq 0$ for $0 \leq x<\infty$.

We now claim that $(j+1 \leq r \leq p)$

$$
\begin{aligned}
\int_{0}^{\infty} & \left(P_{n}^{\prime}(x)\right)^{r-j}\left(P_{n}(x)\right)^{j} x^{p-1} e^{-x} d x \\
& \leq \frac{n}{(n-1) r+p+j} \int_{0}^{\infty}\left(P_{n}^{\prime}(x)\right)^{r-j-1}\left(P_{n}(x)\right)^{j+1} x^{p-1} e^{-x} d x
\end{aligned}
$$


First we note that for $j+1<r \leq p$ we have

$$
\begin{aligned}
I_{r, j}= & \int_{0}^{\infty}\left(P_{n}^{\prime}(x)\right)^{r-j}\left(P_{n}(x)\right)^{j} x^{p-1} e^{-x} d x \\
= & \int_{0}^{\infty}\left(P_{n}^{\prime}(x)\right)^{r-j-2}\left(P_{n}(x)\right)^{j} x^{p-1} e^{-x}\left(\left(P_{n}^{\prime}(x)\right)^{2}-P_{n}(x) P_{n}^{\prime \prime}(x)\right) d x \\
& +\int_{0}^{\infty}\left(P_{n}^{\prime}(x)\right)^{r-j-2}\left(P_{n}(x)\right)^{j+1} x^{p-1} e^{-x} P_{n}^{\prime \prime}(x) d x
\end{aligned}
$$

using (4.2), we have

$$
\begin{aligned}
I_{r, j} \leq & \int_{0}^{\infty}\left(P_{n}^{\prime}(x)\right)^{r-j-1}\left(P_{n}(x)\right)^{j+1} x^{p-2} e^{-x} d x \\
& +\int_{0}^{\infty}\left(P_{n}^{\prime}(x)\right)^{r-j-2}\left(P_{n}(x)\right)^{j+1} x^{p-1} e^{-x} P_{n}^{\prime \prime}(x) d x .
\end{aligned}
$$

Next, we observe that

$$
\begin{aligned}
\int_{0}^{\infty} P_{n}^{\prime \prime}(x)\left(P_{n}^{\prime}(x)\right)^{r-j-2}\left(P_{n}(x)\right)^{j+1} x^{p-1} e^{-x} d x \\
=\frac{1}{r-j-1} \int_{0}^{\infty} \frac{d}{d x}\left(P_{n}^{\prime}(x)\right)^{r-j-1}\left(P_{n}(x)\right)^{j+1} x^{p-1} e^{-x} d x \\
=-\frac{1}{r-j-1} \int_{0}^{\infty}\left(P_{n}^{\prime}(x)\right)^{r-j-1} \\
\quad \times\left\{-e^{-x} x^{p-1}\left(P_{n}(x)\right)^{j+1}+(p-1) x^{p-2} e^{-x}\left(P_{n}(x)\right)^{j+1}\right. \\
\left.\quad+(j+1)\left(P_{n}(x)\right)^{j} P_{n}^{\prime}(x) x^{p-1} e^{-x}\right\} d x .
\end{aligned}
$$

From (4.4) and (4.5) we obtain

$$
\begin{aligned}
I_{r, j} \leq & \int_{0}^{\infty}\left(P_{n}^{\prime}(x)\right)^{r-j-1}\left(P_{n}(x)\right)^{j+1} x^{p-2} e^{-x} d x \\
& +\frac{1}{r-j-1} \int_{0}^{\infty}\left(P_{n}^{\prime}(x)\right)^{r-j-1}\left(P_{n}(x)\right)^{j+1} e^{-x} x^{p-1} d x \\
& -\frac{p-1}{r-j-1} \int_{0}^{\infty}\left(P_{n}^{\prime}(x)\right)^{r-j-1}\left(P_{n}(x)\right)^{j+1} x^{p-2} e^{-x} d x \\
& -\frac{j+1}{r-j-1} I_{r, j} .
\end{aligned}
$$

From above, we obtain

$$
\begin{aligned}
r I_{r, j} \leq & (r-p-j) \int_{0}^{\infty}\left(P_{n}^{\prime}(x)\right)^{r-j-1}\left(P_{n}(x)\right)^{j+1} x^{p-2} e^{-x} d x \\
& +\int_{0}^{\infty}\left(P_{n}^{\prime}(x)\right)^{r-j-1}\left(P_{n}(x)\right)^{j+1} x^{p-1} e^{-x} d x .
\end{aligned}
$$

Next, we note that

$$
\left(P_{n}^{\prime}(x)\right)^{r-j-1}\left(P_{n}(x)\right)^{j+1}=\sum_{k=1}^{(n-1) r+j+1} b_{k} x^{k}, \quad b_{k} \geq 0 .
$$


Therefore,

$$
\begin{aligned}
\int_{0}^{\infty}\left(P_{n}^{\prime}(x)\right)^{r-j-1}\left(P_{n}(x)\right)^{j+1} x^{p-1} e^{-x} d x \\
=\sum_{k=1}^{(n-1) r+j+1} b_{k} \int_{0}^{\infty} x^{k+p-1} e^{-x} d x \\
=\sum_{k=1}^{(n-1) r+j+1} b_{k}(k+p-1) !
\end{aligned}
$$

and

$$
\int_{0}^{\infty}\left(P_{n}^{\prime}(x)\right)^{r-j-1}\left(P_{n}(x)\right)^{j+1} x^{p-2} e^{-x} d x=\sum_{k=1}^{(n-1) r+j+1} b_{k}(k+p-2) !
$$

From these two relations, it follows that

$$
\begin{aligned}
& \int_{0}^{\infty}\left(P_{n}^{\prime}(x)\right)^{r-j-1}\left(P_{n}(x)\right)^{j+1} x^{p-1} e^{-x} d x \\
& \quad \leq((n-1) r+j+p) \int_{0}^{\infty}\left(P_{n}^{\prime}(x)\right)^{r-j-1}\left(P_{n}(x)\right)^{j+1} x^{p-2} e^{-x} d x .
\end{aligned}
$$

Therefore, using (4.8) and (4.6) we obtain

$$
\begin{aligned}
r I_{r, j} & \leq\left(1+\frac{r-p-j}{(n-1) r+j+p}\right) \int_{0}^{\infty}\left(P_{n}^{\prime}(x)\right)^{r-j-1}\left(P_{n}(x)\right)^{j+1} x^{p-1} e^{-x} d x \\
& =\frac{n r}{(n-1) r+j+p} \int_{0}^{\infty}\left(P_{n}^{\prime}(x)\right)^{r-j-1}\left(P_{n}(x)\right)^{j+1} x^{p-1} e^{-x} d x .
\end{aligned}
$$

From above, (4.3) follows, for $j+1<r \leq p$.

The proof of (4.3) for $j+1=r$ is as follows. From (4.8) we have

$$
\int_{0}^{\infty}\left(P_{n}(x)\right)^{r} x^{p-1} e^{-x} d x \leq(n r+p-1) \int_{0}^{\infty}\left(P_{n}(x)\right)^{r} x^{p-2} e^{-x} d x .
$$

Also

$$
\begin{aligned}
\int_{0}^{\infty} & \left(P_{n}^{\prime}(x)\right)^{r-j}\left(P_{n}(x)\right)^{j} x^{p-1} e^{-x} d x \\
& =\int_{0}^{\infty} P_{n}^{\prime}(x)\left(P_{n}(x)\right)^{r-1} x^{p-1} e^{-x} d x \\
& =\frac{1}{r} \int_{0}^{\infty} \frac{d}{d x}\left(P_{n}(x)\right)^{r} x^{p-1} e^{-x} d x \\
& =-\frac{1}{r} \int_{0}^{\infty}\left(P_{n}(x)\right)^{r}\left(-e^{-x} x^{p-1}+(p-1) x^{p-2} e^{-x}\right) d x \\
& \leq\left(\frac{1}{r}-\frac{p-1}{n r+p-1}\right) \int_{0}^{\infty}\left(P_{n}(x)\right)^{r} x^{p-1} e^{-x} d x \\
& =\frac{n}{n r+p-1} \int_{0}^{\infty}\left(P_{n}(x)\right)^{r} x^{p-1} e^{-x} d x .
\end{aligned}
$$


From (4.3), we have $(j+1 \leq r \leq p)$

$$
\begin{aligned}
& \frac{\int_{0}^{\infty}\left(P_{n}^{\prime}(x)\right)^{r} x^{p-1} e^{-x} d x}{\int_{0}^{\infty}\left(P_{n}(x)^{r} x^{p-1} e^{-x} d x\right.} \\
& \quad \leq \frac{n^{r}}{[(n-1) r+p][(n-1) r+p+1] \cdots[(n-1) r+p+r-1]} \\
& \quad \leq \frac{n^{r}(n r+p-r-1) !}{(n r+p-1) !}
\end{aligned}
$$

((4.9) become an equality for $\left.P_{n}(x)=\alpha x^{n}\right)$.

This proves Theorem 3 as well.

\section{ACKNOWLEDGMENT}

The author wishes to thank the referee for many valuable suggestions.

\section{REFERENCES}

1. J. Eröd, Bizonyos polinomok maximumáról, Mat. Fiz. Lapok 46 (1939), 58-82.

2. P. Erdös, Extremal properties of derivatives of polynomials, Ann. of Math. 2 (1940), 310-313.

3. E. Hille, G. Szegö, and J. D. Tamarkin, On some generalizations of a theorem of A. A. Markoff, Duke Math. J. 3 (1937), 729-739.

4. G. G. Lorentz, Derivatives of polynomials with positive coefficients, J. Approx. Theory 1 (1968), 1-4.

5. J. Szabados and A. K. Varma, Inequalities for derivatives of polynomials having real zeros, Approximation Theory III, The University of Texas, Austin (E. W. Cheney, eds.), Academic Press, New York, 1980, pp. 881-888.

6. G. Szegö, On some properties of approximation, Magyar Tud. Akad. Mat. Kutató Int. Közl. 2 (1964), 3-9.

7. P. Turán, Über die Ableitung von Polynomen, Compositio Math. (1939), 89-95.

8. A. K. Varma, Some inequalities of algebraic polynomials having all zeros inside $[-1,1]$, Proc. Amer. Math. Soc. 88 (1983), 227-233.

9. $\ldots$ Derivative of polynomials with positive coefficients, Proc. Amer. Math. Soc. 83 (1981), 107-112.

Department of Mathematics, University of Florida, Gainesville, Florida 32611 